\title{
PREDICTING LANDFALL's LOCATION AND TIME OF A Tropical Cyclone USING REANALysis Data
}

\author{
Sandeep Kumar \\ Department of Mathematics, Shaheed Bhagat Singh College, \\ University of Delhi. \\ $\&$ \\ Department of Computer Science, IIIT Delhi \\ New Delhi, India. \\ sandeep_kumar@sbs.du.ac.in, sandeepk@iitt.ac.in
}

\author{
Koushik Biswas \\ Department of Computer Science \\ IIIT Delhi \\ New Delhi, India, 110020 \\ koushikb@iiitd.ac.in
}

\author{
Ashish Kumar Pandey \\ Department of Mathematics \\ IIIT Delhi \\ New Delhi, India, 110020 \\ ashish.pandey@iiitd.ac.in
}

\begin{abstract}
Landfall of a tropical cyclone is the event when it moves over the land after crossing the coast of the ocean. It is important to know the characteristics of the landfall in terms of location and time, well advance in time to take preventive measures timely. In this article, we develop a deep learning model based on the combination of a Convolutional Neural network and a Long Short-Term memory network to predict the landfall's location and time of a tropical cyclone in six ocean basins of the world with high accuracy. We have used high-resolution spacial reanalysis data, ERA5, maintained by European Center for Medium-Range Weather Forecasting (ECMWF). The model takes any 9 hours, 15 hours, or 21 hours of data, during the progress of a tropical cyclone and predicts its landfall's location in terms of latitude and longitude and time in hours. For 21 hours of data, we achieve mean absolute error for landfall's location prediction in the range of $66.18-158.92$ kilometers and for landfall's time prediction in the range of $4.71-8.20$ hours across all six ocean basins. The model can be trained in just 30 to 45 minutes (based on ocean basin) and can predict the landfall's location and time in a few seconds, which makes it suitable for real time prediction.
\end{abstract}

Keywords Tropical cyclone $\cdot$ Landfall $\cdot$ Reanalysis data

\section{Introduction}

Predicting natural disasters is one of the difficult prediction problems because of the complex interplay between various cause factors, which vary with space and time. One such natural disaster is a Tropical Cyclone (TC) that frequently occurs in tropical and subtropical regions of the world. TCs are also called Hurricanes or Typhoons in different parts of the world. TCs are characterized by low-pressure areas with an atmospheric circulation that brings heavy rainfall, strong winds, thunderstorms, and flash floods in the coastal regions, thereby affecting human lives, property, transportation, businesses, and society. Each year TCs are responsible for the deaths of hundreds of people and billions of economic losses [1, 2]. For example, recent cyclones Willa (2018) in the East Pacific (EP) ocean, Yutu (2018) in the East Pacific (EP) ocean, Harold (2019) in the South Pacific (SP) ocean, Amphan (2020) in the North Indian ocean, Marcus (2018) in the South Indian(SI) and Michael (2018) in North Atlantic ocean caused economic losses of nearly US $\$ 41$ billion in total. 
The most important event during the progress of a TC is its landfall that is when it reaches land after crossing the coast of the ocean. The economic and human losses caused by a TC are centered around few kilometers of its landfall location. Therefore, it is crucial to predict the location and time of the landfall of a TC well advance in time with high accuracy. When a TC is over the ocean, the TC's real-time data may not be continuously available because of measurement constraints. Therefore, the landfall prediction model must be flexible regarding the data it requires for prediction. The model should pick continuous data from the course of the TC whenever available and provide predictions of landfall's location and time with high accuracy. In this study, we have used a deep learning model to predict the landfall's location and time of a TC. The model takes 9 hours (h), 15h, or $21 \mathrm{~h}$ of continuous data, anytime during the course of a TC, and predicts the landfall's location and time at least $12 \mathrm{~h}$ before the actual landfall time. The model performance is reported for six ocean basins - North Indian (NI), South Indian (SI), West Pacific (WP), East Pacific (EP), South Pacific (SP), and North Atlantic (NA) oceans. As per our knowledge, this is the first work that directly focuses on predicting the characteristics of the landfall of a TC using reanalysis data. Predicting landfall's characteristics is a challenging problem to deal with, as discussed in [3].

There are numerous existing TC track prediction models that can be classified into numerical (or dynamical) models, statistical models, and ensemble models [4]. The numerical models rely on physical equations governing atmospheric circulations to capture the evolution of the atmospheric fields. These methods are computationally involved and need large supercomputers. The few major operational numerical models for track prediction are - European Center for Medium-Range Weather Forecasts (ECMWF), Global Forecast System (GFS), Hurricane Weather and Research Forecasting model (HWRF), and Hurricane Multi-scale Ocean-coupled Non-hydrostatic model (HMON). The statistical models [5] do not require high computational resources and rely on finding a relationship between historical data and cyclone specific features. The primary operational, statistical models for track prediction are - Climatology and Persistence model (CLIPER5) and Trajectory-CLIPER. The ensemble models combine numerical and statistical models for prediction. Generally, ensemble models perform better than individual models [6]. Numerical methods and statistical methods have their own limitations, and we need to make a trade-off between computational cost and capturing of the complex relationship between various cause factors.

Recently, with the increase in the data related to tropical cyclones, various studies have appeared that have successfully applied machine learning-based models to predict various characteristics of a tropical cyclone [3, 7] which we discuss in detail in the next section. The article is organized as follows: Section 2 describes the related work in this direction, Section 3 describes the data used in this study, Section 4 describes the deep learning model used to forecast the above mentioned prediction problem. Finally, in Section 5 , we present the results and analysis of our model. In Section 6 , we conclude and provide future directions.

\section{Related Work}

Initial studies regarding tropical cyclones track and intensity forecasts used Artificial Neural Networks (ANNs) [8, 9]. Since the prediction problems related to atmospheric conditions involve both spatial and temporal components, deep learning models like Recurrent Neural Networks (RNNs), Long Short-Term Memory (LSTM) networks, Convolutional Neural Networks (CNNs), and combinations of these have been successfully deployed to capture the complex non-linear interplay between various atmospheric components both of spatial and temporal nature. In [7], sparse RNN with flexible topology is used for the prediction of hurricane trajectory in the Atlantic ocean. In [10], ConvLSTM is used to predict the hurricane trajectory from the past density maps of hurricane trajectories. In [11], authors have presented a fully connected RNN model to predict cyclone trajectories from historical cyclone data in the Atlantic ocean. In [12], a nowcasting model is presented based on an LSTM network to predict typhoon trajectory.

Recently, few studies have dealt with TC formation, track, and intensity prediction problems using reanalysis data [13, 14, 15]. In [15], reanalysis dataset has been used to forecast typhoon formation forecasting in NA, EP, and WP oceans. In [13], authors have used historical data of a TC along with reanalysis data from ERA-Interim [16] to predict the track of TC with a lead time of $24 \mathrm{~h}$ in six ocean basins. They propose a fusion network in which the output of CNN trained on wind fields and pressure fields from reanalysis data and output of an ANN trained on historical TC data are fed into another ANN network. Their model does not take the temporal aspect into account as they have stacked input from two-time steps $t$ and $t-6$ to feed into a CNN. In [14], TC intensity and track prediction task is achieved with a lead time of 24h, using reanalysis data ERA5 [17], historical TC data, and output from operational forecast models for NA and EP ocean basins. They have proposed framework Hurricast (HURR) consisting of seven different models that used different combinations of CNN, GRU, Transformers, and XGBoost models. They used data between $t$ to $t-21$ hours and capture the spatial and temporal aspects of data, thereby addressing the shortcoming of [13]. As there is no existing work that predicts the landfall characteristics from reanalysis and historical TC data, we will try to compare our model with the models presented in [13, 14]. 


\section{Data}

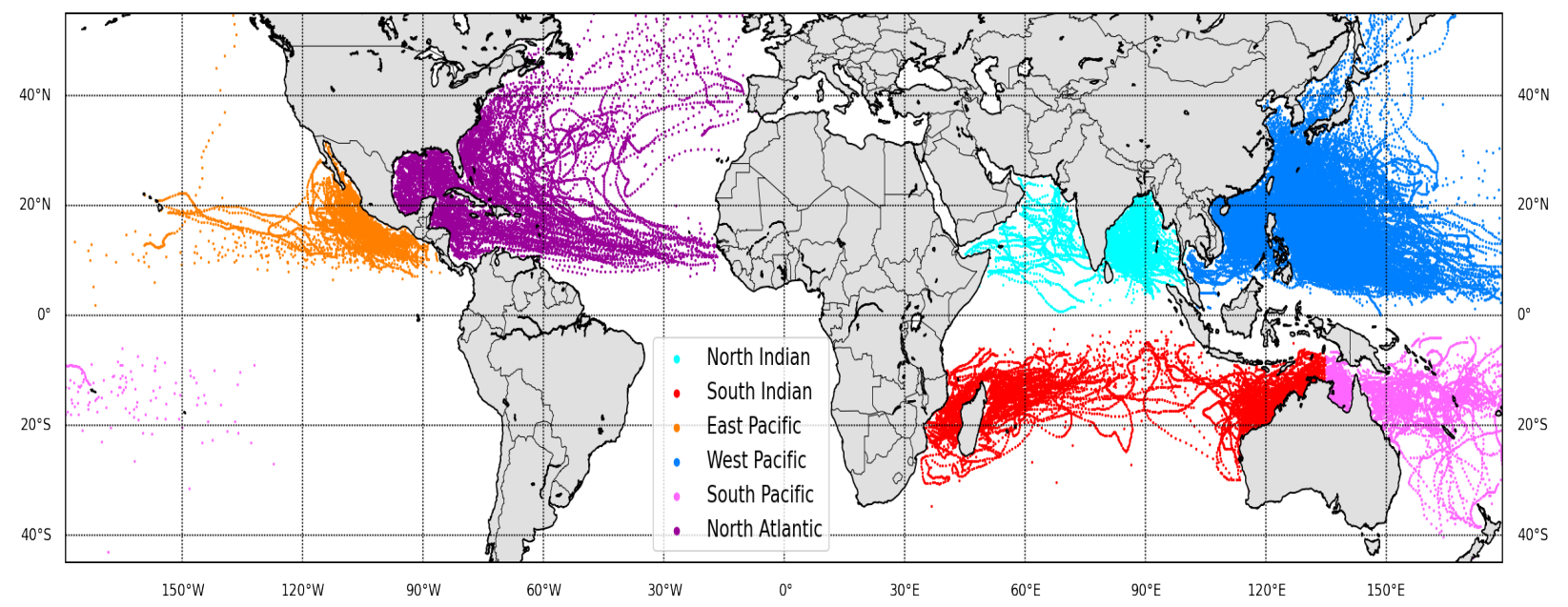

Figure 1: Trajectory of all cyclones till landfall in six ocean basins

In this study, we have used two open-source datasets - historical cyclones data and reanalysis data. The historical track data is taken from NOAA database IBTrACS version 4 [18, 19]. The dataset contains information about ocean basin, latitude, longitude, Estimated Central Pressure (ECP), Maximum Sustained surface Wind Speed(MSWS), distance to land, distance and direction of TC movement, etc at an interval of 3hours. From these features, we choose latitude, longitude, and distance to land for our study and exclude features like MSSW, ECP, distance, and direction of TC movement, which are used by two related works [13, 14]. As we are predicting the landfall's location and time of a TC, our dataset consists of all those cyclones which hit the coastal region, and for all such cyclones, only data corresponding to the time points when a TC was moving over the ocean is taken into account. If a cyclone moves from ocean to land and then from land to ocean and continues like this during its course, then the data corresponding to its presence over land is not considered. Also, if a cyclone moves from ocean to land and land to ocean multiple times, then each such movement is treated as a separate cyclone while preparing the dataset. Because of this process, our dataset size is shortened. We are not using features like MSSW, ECP, distance, and direction of TC movement, which have lots of missing values to avoid further shortening of our dataset. We extracted data from 1981 to 2020 provided at an interval of three hours. The trajectory of all TCs till their landfall considered in this study are shown in Figure 1

The large scale atmospheric circulation of wind at different pressure levels plays a crucial role in determining the track of a TC. To capture this information, we have used ERA5 [17] reanalysis data produced by ECMWF in near to real-time. ERA5 is a fifth-generation reanalysis data covering global climate and weather since 1950. From 1979 onwards, the high-resolution data ERA5 replaces the ERA-interim. Reanalysis is a scientific way of producing globally complete and consistent data by gathering the information from various resources and validating them using the laws of physics. We extracted the $u, v$ components of wind and $z$ geopotential fields at three atmospheric pressure levels $(225 \mathrm{hPa}, 500 \mathrm{hPa}$, $700 \mathrm{hPa}$ ) for a spatial extension of $4 \times 4$ degree and spatial resolution of $0.25 \times 0.25$ degrees (resulting in a grid of size $33 \times 33$ ), centered at the current TC location. The $u$ and $v$ component of wind represents its eastward-westward and northward-southward movement respectively. The $z$ geopotential represents gravitational potential energy of a unit mass relative to sea level. The choice of these variables is inspired by [13, 14] where authors have used values of these three variables at mentioned three pressure levels for a spatial extension of $25 \times 25$ degrees and spatial resolution of 1 $\times 1$ degree (resulting in a grid of size $25 \times 25$ ). On the world map, one degree approximately equals $110 \mathrm{kilometers}$ (KM). This way, we are utilizing the mentioned variables values for a spatial spread of around 440 KM (in comparison of $1320 \mathrm{KM}$ by earlier two studies) with a spatial resolution of around $27.5 \mathrm{KM}$ (in comparison of $110 \mathrm{KM}$ by earlier two studies) around the TC center. Apart from these three variables, we extracted the sea surface temperature (SST) for the $33 \times 33$ grid centered at the TC location for each time point. A pictorial depiction of reanalysis data is shown in Figure 2 .

As we are using CNN, to feed the current location information, we have created two more $33 \times 33$ matrices lats and longs for each time point of a TC. The each row of lats is equal to vector $($ lat $+0.25 * k \mid-16 \leq k \leq 16)$ and each column of longs is equal to vector (long $+0.25 * k \mid-16 \leq k \leq 16$ ) where (lat, long) denotes the latitude and longitude of TC's current location. Feeding this information in $\overline{C N N}$ will enable it to generate distance and direction like features between two successive time points of a TC. 


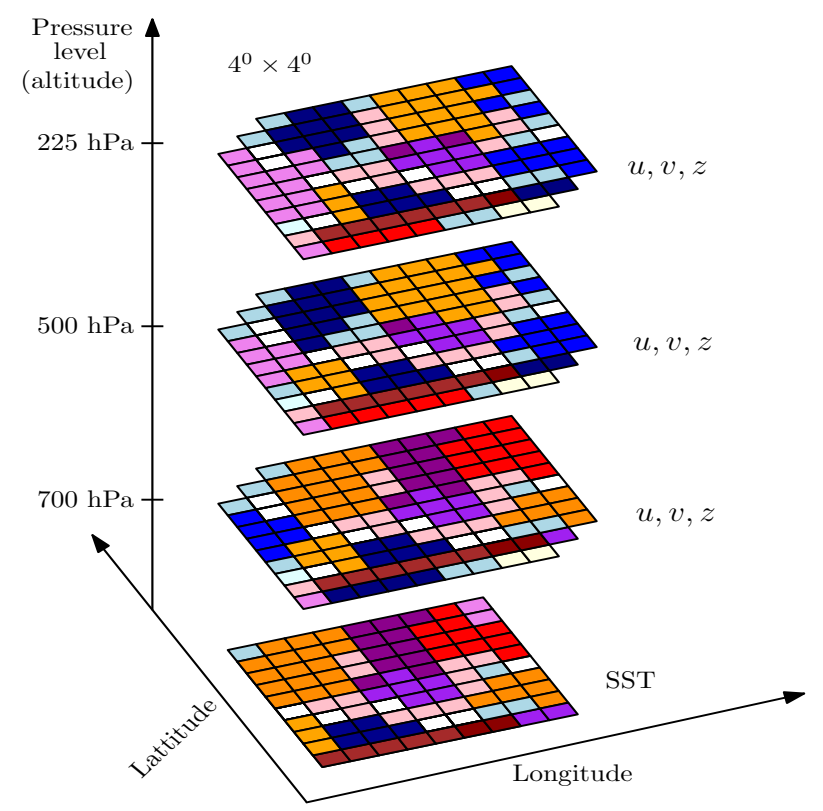

Figure 2: A pictorial depiction of $\mathrm{u}, \mathrm{v}, \mathrm{z}$ wind fields and SST

Table 1: Dataset size and Landfall time (hours)

\begin{tabular}{|l|l|l|l|l|l|}
\hline $\begin{array}{l}\text { Ocean } \\
\text { Basin }\end{array}$ & $\begin{array}{l}\text { No. of } \\
\text { TCs }\end{array}$ & $\begin{array}{l}\text { Size of } \\
\text { Dataset }\end{array}$ & $\begin{array}{l}\text { Average } \\
\text { Time }\end{array}$ & $\begin{array}{l}\text { Min } \\
\text { Time }\end{array}$ & $\begin{array}{l}\text { Max } \\
\text { Time }\end{array}$ \\
\hline NI & 205 & 5920 & 95.61 & 21 & 270 \\
\hline SI & 282 & 10600 & 121.74 & 21 & 516 \\
\hline EP & 116 & 4000 & 112.44 & 21 & 315 \\
\hline SP & 189 & 5674 & 99.06 & 21 & 513 \\
\hline WP & 1064 & 39166 & 119.43 & 21 & 606 \\
\hline NA & 401 & 11386 & 94.18 & 21 & 531 \\
\hline
\end{tabular}

In Table 1 the dataset size along with average landfall time after the initiation of a TC in six ocean basins are shown for all TCs with the minimum time difference between TC formation and its landfall as $21 \mathrm{~h}$.

\subsection{Training Dataset Preparation}

Let $T$ be the number of continuous data points (that is $3(T-1)$ hours of data) taken in the model to predict the target. For a fixed cyclone, let $T_{L}$ be the number of data points between cyclone formation and its landfall. For this TC, we created $T_{L}-(T-1)-3=T_{L}-T-2$ inputs, where a single input is a sequence of $T$ vectors of the form:

$$
(\operatorname{lats}(t), \operatorname{longs}(t), \mathrm{u} 225(t), \mathrm{v} 225(t), \mathrm{z} 225(t), \mathrm{u} 500(t), \mathrm{v} 500(t), \mathrm{z} 500(t), \mathrm{u} 700(t), \mathrm{v} 700(t), \mathrm{z} 700(t), \operatorname{SST}(t))
$$

where $k \leq t \leq T+k-1$ and $k$ varies from 1 to $T_{L}-T-2$. The target variables for each input are latitude and longitude at landfall or time (in hours) remaining to landfall of the cyclone from the current time $t$. One must note that by following the above process, we are predicting our target at least $12 \mathrm{~h}$ before the landfall. For example, BELNA cyclone formed at 00 hours on 05 December 2019 in EP ocean, and the landfall happened at 15 hours on 09 December 2019 , that is $T_{L}=37$. Suppose $T=8$, then this TC will generate $37-8-2=27$ training data points. The collection of all such inputs across all TCs for a particular ocean basin will form the training dataset.

\section{Model Implementation and Training}

As we are dealing with a dataset with both spatial and temporal dimensions, we have used a combination of CNN and LSTM models. In this section we will briefly describe the ANN, RNN, LSTM and CNN models. 


\subsection{Artificial Neural Network}

ANNs [20] are connected networks of layers, where each layers consists of nodes. Generally the nodes in one layer are connected to nodes in succeeded and preceded layers. Each such connection is assigned weights, that regulates the information flow between nodes from one layer to another layer. The information flow at one node is a composition of a non-linear function with weighted linear sum of incoming connections. The non-linear function is called an activation function, which helps in learning non-linear relationship between input and output variables. The weights assigned to each connection is updated in a way to minimize the suitably chosen loss function through the Gradient Descent algorithm [21]. The intermediate layers between the input and output layers are called hidden layers. A fully connected ANN with two hidden layers is shown in Figure 3

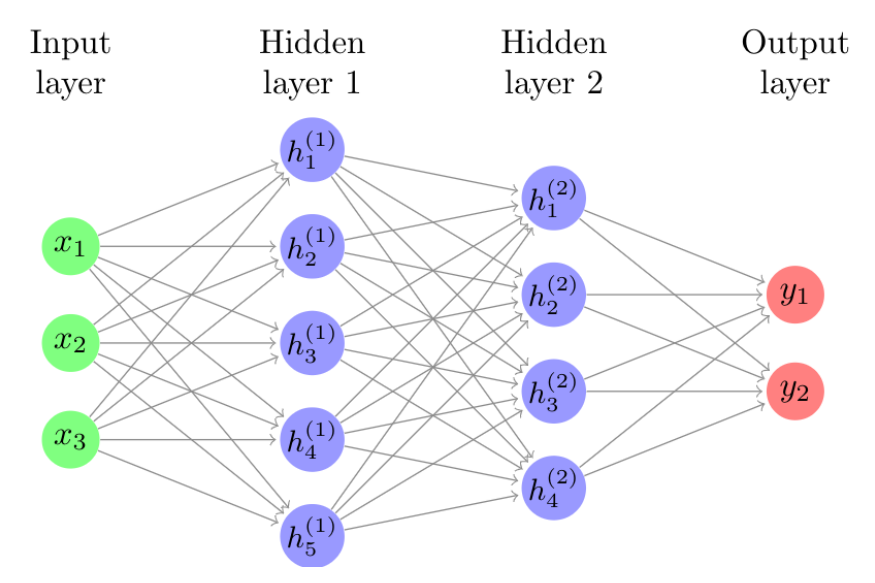

Figure 3: A fully connected ANN

Mathematically, the information flow in an ANN can be represented as $h_{j}^{(i)}=\sigma\left(W_{j}^{(i)} X\right)$, where $X$ is the input matrix containing bias, $h_{j}^{(i)}$ represents the information at the $j^{\text {th }}$ neuron in the $i^{\text {th }}$ layer, $W_{j}^{i}$ is weight matrix that determines interaction between nodes at $(i-1)^{t h}$ and $i^{t h}$ layer, and $\sigma$ is the activation function. ANN models are not best choice for time-series data, where the output is a function of sequence of inputs. RNN models are best suited for time-series data that has ability of information persistence.

\subsection{Recurrent Neural Network}

Recurrent neural networks (RNNs) [22, 23, 24] are like ANNs with internal connections. They are suitable for time series data as they can remember information from inputs at past time points along with current input. A RNN structure is described in Figure 4. Mathematically, the information flow in a RNN can be represented as

$$
h_{t}=\sigma\left(W_{h} x_{t}+U_{h} h_{t-1}+b_{h}\right), \quad y_{t}=\sigma\left(W_{y} h_{t}+b_{y}\right)
$$

where $x_{t}$ is the input, $h_{t}$ is the hidden state, $y_{t}$ is the output at time $t, W_{h}, W_{y}$ and $U_{h}$ are weight matrices, $b_{h}, b_{y}$ are the biases, and $\sigma$ is the activation function.

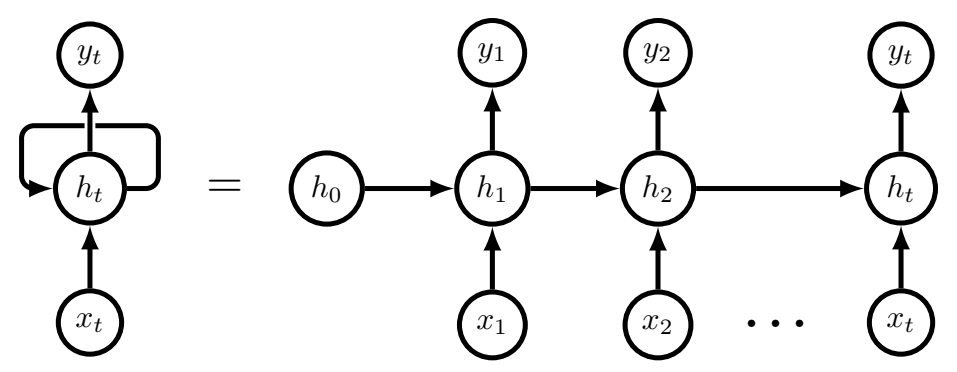

Figure 4: Structure of RNN 
However, they suffer from the problem of exploding and vanishing gradients [25]. LSTM networks [26, 27, 28, 29] overcomes this problem by maintaining three inner cell gates and a memory cell to handle past dependencies. LSTM are successfully applied on TCs track and intensity related prediction problems in [11, 12, 7].

\subsection{Convolutional Neural Network (CNN)}

In recent times, CNNs [30, 31, 32] have become the default choice to deal with spatial data like satellite images or image-like data. They are inspired by the cortex visual system, where each neuron process data of its neighboring fields. Like an ANN, CNN also have an input layer, an output layer, and multiple hidden layers consisting of convolutional layers, pooling layers, and fully connected layers. The convolution operation shares the weight across the input that enables a CNN to learn features with a small number of parameters. Pooling is a downsampling method that helps to reduce the dimensions of the feature maps. Pooling helps to manage overfitting, reduce the computational cost by decreasing the number of weights in the model. CNNs are successfully applied to solving atmospheric dynamical phenomenons. In [33, 10] authors have used CNN to predict the TCs trajectories. In [34], physical processes knowledge is combined with CNN to predict the SST. In [35, 15] CNNs are used to predict the formation and intensity of a TC.

\subsection{Model Description}

We have used a combination of CNN and LSTM to capture the spatial and temporal aspects of our prediction problem. We come up with a model that works well for each ocean basin. The structure of the model for location prediction in terms of latitude and longitude is described in the Figure 5 for $T=8$. The model for time prediction is the same except that the last dense layer's output size is one. The model is implemented in Keras API [36] which uses underlying low-level language TensorFlow [37]. For $T=8$, one training point consists of eight sequential inputs of shape $12 \times 33 \times 33$, where 12 represents the number of channels (nine channels of wind and geopotential fields, one of SST, two of latitudes and longitudes). We extracted the features corresponding to each time point of the input using the TimeDistributed layer of Keras, which are further fed into LSTM layers as shown in Figure 5.

\subsection{Training}

For training purposes, the dataset is divided into three parts training, validation, and test set in the ratio of 60:20:20. We tried different configurations and hyperparameters for our model and choose the one that gives the best result on validation data after taking the issue of overfitting into account. Finally, we have reported the 5-fold test accuracy of our model. We trained the model for $T$ equals to 4,6 , and 8 ; increasing $T$ further does not lead to improved accuracy. The input features are scaled using Standard Scaler of Scikit learn library [38], which is given by $f(x)=\frac{x-\mu}{\sigma}$, where $\mu$ is the mean and $\sigma$ is the standard deviation. The target variables are also scaled in case of landfall's latitude and longitude prediction but not for time prediction. The model uses the mean square error (MSE) as the loss function. We have reported the model performance in terms of root mean square error (RMSE) and mean absolute error (MAE), which are defined as follows:

$$
\mathrm{MSE}=\frac{1}{n} \sum_{i=1}^{n}\left(y_{i}-\bar{y}_{i}\right)^{2}, \quad \mathrm{RMSE}=\sqrt{M S E}, \quad \mathrm{MAE}=\frac{1}{n} \sum_{i=1}^{n}\left|y_{i}-\bar{y}_{i}\right|,
$$

where $y_{i}$ is the actual value and $\bar{y}_{i}$ is the predicted value. The model uses the Adam [39] optimizer with a default learning rate 0.001 to minimize the loss function. Convolution layers and LSTM layers uses the activation function $\operatorname{ReLU}(x)=\max (0, x)$ [40]. The model uses a total of 100 epochs. We run our experiments on Nvidia Tesla V100 GPU with 16 GB RAM. The model takes approximately 30 to 45 minutes (depending on the ocean basin) to complete 100 epochs.

\section{Results and Comparison}

Our model, at any time $t$ during the progress of a TC, takes $T$ number $((T-1) * 3 \mathrm{~h})$ of data and predicts the landfall's latitude, longitude, and time. For example, if $T=8$ and a particular TC is at the $t=42 h$ during its progression, then using the data between the time $t=42-21=21$ and $t$. the model predicts the landfall's characteristics. To avoid any bias when time $t$ is very close to landfall's time, the model predicts only for $t \leq L-12$, where $L$ is the landfall's time, which means we are remaining at least 12 hours away from landfall while predicting. For each ocean basin and for different values of $T=4,6,8$ ( $9 \mathrm{~h}, 15 \mathrm{~h}, 21 \mathrm{~h})$. we have reported the size of the dataset and the 5-fold accuracy on the test dataset in terms of RMSE and MAE along with standard deviation in Table 2 . From the predicted latitude and longitude, the distance error between actual landfall location and predicted location is also reported in Table 2 


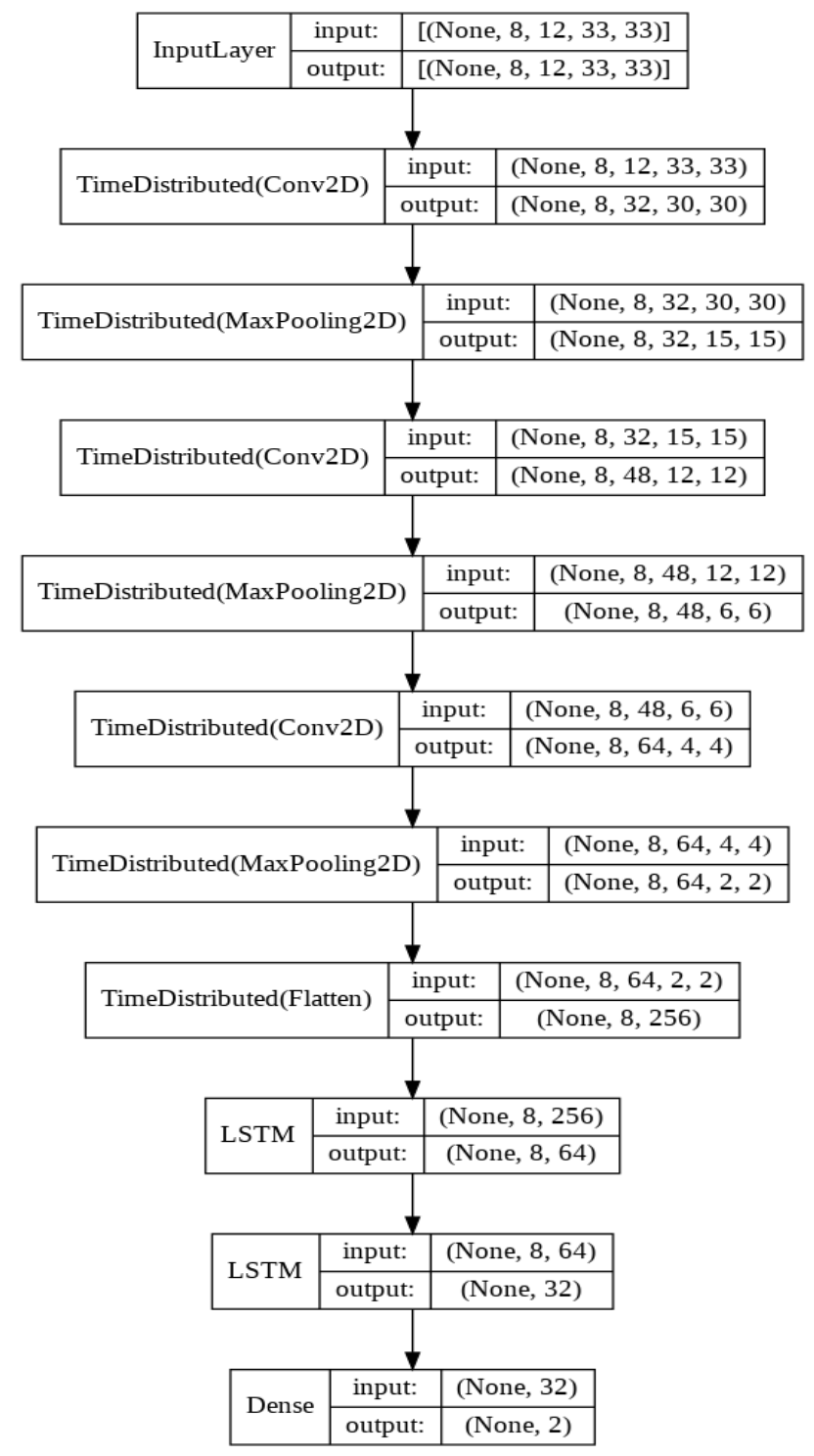

Figure 5: Model representation for latitude/longitude prediction for $T=8$

From Table 2 we can see that we can predict the landfall's location in ocean basins NI, SI, EP, SP, WP, and NA with a distance error of $66.18 \mathrm{KM}, 119.96 \mathrm{KM}, 110.48 \mathrm{KM}, 144.80 \mathrm{KM}, 108.0 \mathrm{KM}$, and $158.92 \mathrm{KM}$ respectively for $T=8(21 \mathrm{~h})$ with a low standard deviation (std). If we look at the landfall's time prediction results, they are quite impressive. The model predicts the landfall's time in six ocean basins NI, SI, EP, SP, WP, and NA with an MAE of 4.71h, 6.04h, 8.20h, $6.74 \mathrm{~h}, 5.89 \mathrm{~h}$, and $7.42 \mathrm{~h}$ respectively with low standard deviation.

To further demonstrate the working of our model, in Figures 6 and 7, we have shown landfall's latitude-longitude prediction results for two cyclones BUD (2018) and BELNA (2019) in ocean basins EP and SI, respectively. In Figures 8 and 9 , the landfall's time prediction results for TCs FANI (2019) and NAKRI (2019) in ocean basins NI and WP, respectively, are shown. One can note that the model start predicting at time $t=21$ and uses data of 21 hours between $t-21$ and $t$ to predict at time $t$. All these named cyclones are not part of training data.

\subsection{Comparison}

As per our knowledge, there does not exist any earlier work that predicts the landfall's location and time using reanalysis data. In the absence of directly related work, we will compare our results with closely related works [13, 14]. In Table 3 , the compassion between Fusion model [13] and our proposed model is shown. In [13] authors used TC data for 12 
Table 2: 5-fold accuracy on Test data of landfall's location and time prediction for different $T$

\begin{tabular}{|c|c|c|c|c|c|c|c|c|c|}
\hline \multirow{2}{*}{$\begin{array}{l}\text { Ocean } \\
\text { Basin }\end{array}$} & \multirow{2}{*}{$\begin{array}{l}\text { T } \\
\text { (hours) }\end{array}$} & \multirow{2}{*}{$\begin{array}{l}\text { Dataset } \\
\text { Size }\end{array}$} & \multicolumn{3}{|c|}{ RMSE (std) } & \multicolumn{4}{|c|}{ MAE (std) } \\
\hline & & & $\begin{array}{l}\text { Lati } \\
\text { degree }\end{array}$ & $\begin{array}{l}\text { Long } \\
\text { degree }\end{array}$ & $\begin{array}{l}\text { Time } \\
\text { hours }\end{array}$ & $\begin{array}{l}\text { Lati } \\
\text { degree }\end{array}$ & $\begin{array}{l}\text { Long } \\
\text { degree }\end{array}$ & $\begin{array}{l}\text { Time } \\
\text { hours }\end{array}$ & $\begin{array}{l}\text { Distance } \\
\mathrm{KM}\end{array}$ \\
\hline \multirow{3}{*}{$\begin{array}{l}\text { North } \\
\text { Indian }\end{array}$} & $4(9)$ & 5060 & $\begin{array}{l}0.58 \\
(0.05)\end{array}$ & $\begin{array}{l}1.03 \\
(0.09)\end{array}$ & $\begin{array}{l}10.46 \\
(1.40)\end{array}$ & $\begin{array}{l}0.38 \\
(0.02)\end{array}$ & $\begin{array}{l}0.70 \\
(0.05)\end{array}$ & $\begin{array}{l}7.33 \\
(1.50)\end{array}$ & $\begin{array}{l}93.78 \\
(6.71)\end{array}$ \\
\hline & $6(15)$ & 4660 & $\begin{array}{l}0.53 \\
(0.13)\end{array}$ & $\begin{array}{l}0.90 \\
(0.13)\end{array}$ & $\begin{array}{l}8.98 \\
(3.47)\end{array}$ & $\begin{array}{l}0.36 \\
(0.12)\end{array}$ & $\begin{array}{l}0.59 \\
(0.10)\end{array}$ & $\begin{array}{l}6.05 \\
(2.38)\end{array}$ & $\begin{array}{l}81.92 \\
(18.62)\end{array}$ \\
\hline & $8(21)$ & 4284 & $\begin{array}{l}0.40 \\
(0.04)\end{array}$ & $\begin{array}{l}0.76 \\
(0.06)\end{array}$ & $\begin{array}{l}6.72 \\
(0.58)\end{array}$ & $\begin{array}{l}0.26 \\
(0.02)\end{array}$ & $\begin{array}{l}0.50 \\
(0.03)\end{array}$ & $\begin{array}{l}4.71 \\
(0.54)\end{array}$ & $\begin{array}{l}66.18 \\
(2.87)\end{array}$ \\
\hline \multirow{3}{*}{$\begin{array}{l}\text { South } \\
\text { Indian }\end{array}$} & 4 4(9) & 9441 & $\begin{array}{l}0.53 \\
(0.03)\end{array}$ & $\begin{array}{l}1.78 \\
(0.22)\end{array}$ & $\begin{array}{l}13.17 \\
(0.59)\end{array}$ & $\begin{array}{l}0.36 \\
(0.02)\end{array}$ & $\begin{array}{l}1.31 \\
(0.15)\end{array}$ & $\begin{array}{l}8.32 \\
(0.31)\end{array}$ & $\begin{array}{l}150.78 \\
(15.55)\end{array}$ \\
\hline & $6(15)$ & 8886 & $\begin{array}{l}0.46 \\
(0.03)\end{array}$ & $\begin{array}{l}1.44 \\
(0.06)\end{array}$ & $\begin{array}{l}11.34 \\
(0.84)\end{array}$ & $\begin{array}{l}0.30 \\
(0.03)\end{array}$ & $\begin{array}{l}1.06 \\
(0.04)\end{array}$ & $\begin{array}{l}7.24 \\
(0.42)\end{array}$ & $\begin{array}{l}123.32 \\
(5.24)\end{array}$ \\
\hline & $8(21)$ & 8353 & $\begin{array}{l}0.42 \\
(0.02)\end{array}$ & $\begin{array}{l}1.42 \\
(0.18)\end{array}$ & $\begin{array}{l}9.63 \\
(0.96)\end{array}$ & $\begin{array}{l}0.27 \\
(0.03)\end{array}$ & $\begin{array}{l}1.05 \\
(0.10)\end{array}$ & $\begin{array}{l}6.04 \\
(0.45)\end{array}$ & $\begin{array}{l}119.96 \\
(12.22)\end{array}$ \\
\hline \multirow{3}{*}{$\begin{array}{l}\text { East } \\
\text { Pacific }\end{array}$} & $\overline{~ 4(9)}$ & 3505 & $\begin{array}{l}0.70 \\
(0.23)\end{array}$ & $\begin{array}{l}1.49 \\
(0.33)\end{array}$ & $\begin{array}{l}10.40 \\
(1.03)\end{array}$ & $\begin{array}{l}0.52 \\
(0.18)\end{array}$ & $\begin{array}{l}1.08 \\
(0.24)\end{array}$ & $\begin{array}{l}7.17 \\
(0.80)\end{array}$ & $\begin{array}{l}133.43 \\
(33.42)\end{array}$ \\
\hline & $6(15)$ & 3276 & $\begin{array}{l}0.62 \\
(0.04)\end{array}$ & $\begin{array}{l}1.33 \\
(0.24)\end{array}$ & $\begin{array}{l}9.89 \\
(2.17)\end{array}$ & $\begin{array}{l}0.46 \\
(0.03)\end{array}$ & $\begin{array}{l}0.95 \\
(0.10)\end{array}$ & $\begin{array}{l}6.99 \\
(1.74)\end{array}$ & $\begin{array}{l}117.8 \\
(9.76)\end{array}$ \\
\hline & $8(21)$ & 3056 & $\begin{array}{l}0.52 \\
(0.03)\end{array}$ & $\begin{array}{l}1.26 \\
(0.16)\end{array}$ & $\begin{array}{l}11.28 \\
(3.69)\end{array}$ & $\begin{array}{l}0.37 \\
(0.02)\end{array}$ & $\begin{array}{l}0.93 \\
(0.03)\end{array}$ & $\begin{array}{l}8.20 \\
(2.96)\end{array}$ & $\begin{array}{l}110.48 \\
(2.86)\end{array}$ \\
\hline \multirow{3}{*}{$\begin{array}{l}\text { South } \\
\text { Pacific }\end{array}$} & 4 4(9) & 4885 & $\begin{array}{l}0.89 \\
(0.09)\end{array}$ & $\begin{array}{l}2.12 \\
(0.14)\end{array}$ & $\begin{array}{l}17.44 \\
(2.70)\end{array}$ & $\begin{array}{l}0.55 \\
(0.05)\end{array}$ & $\begin{array}{l}1.50 \\
(0.14)\end{array}$ & $\begin{array}{l}11.30 \\
(2.19)\end{array}$ & $\begin{array}{l}179.03 \\
(17.26)\end{array}$ \\
\hline & $6(15)$ & 4520 & $\begin{array}{l}0.89 \\
(0.19)\end{array}$ & $\begin{array}{l}2.30 \\
(0.57)\end{array}$ & $\begin{array}{l}13.24 \\
(1.60)\end{array}$ & $\begin{array}{l}0.57 \\
(0.14)\end{array}$ & $\begin{array}{l}1.57 \\
(0.33)\end{array}$ & $\begin{array}{l}7.69 \\
(0.65)\end{array}$ & $\begin{array}{l}188.81 \\
(37.51)\end{array}$ \\
\hline & $8(21)$ & 4182 & $\begin{array}{l}0.72 \\
(0.10)\end{array}$ & $\begin{array}{l}1.67 \\
(0.23)\end{array}$ & $\begin{array}{l}10.07 \\
(1.77)\end{array}$ & $\begin{array}{l}0.44 \\
(0.07)\end{array}$ & $\begin{array}{l}1.23 \\
(0.20)\end{array}$ & $\begin{array}{l}6.74 \\
(1.04)\end{array}$ & $\begin{array}{l}144.80 \\
(22.44)\end{array}$ \\
\hline \multirow{3}{*}{$\begin{array}{l}\text { West } \\
\text { Pacific }\end{array}$} & 4 4(9) & 34874 & $\begin{array}{l}1.34 \\
(0.34)\end{array}$ & $\begin{array}{l}1.72 \\
(0.37)\end{array}$ & $\begin{array}{l}10.84 \\
(0.30)\end{array}$ & $\begin{array}{l}0.88 \\
(0.24)\end{array}$ & $\begin{array}{l}1.15 \\
(0.26)\end{array}$ & $\begin{array}{l}7.44 \\
(0.41)\end{array}$ & $\begin{array}{l}164.17 \\
(40.08)\end{array}$ \\
\hline & $6(15)$ & 32777 & $\begin{array}{l}1.04 \\
(0.16)\end{array}$ & $\begin{array}{l}1.40 \\
(0.22)\end{array}$ & $\begin{array}{l}10.10 \\
(2.14)\end{array}$ & $\begin{array}{l}0.72 \\
(0.13)\end{array}$ & $\begin{array}{l}0.97 \\
(0.16)\end{array}$ & $\begin{array}{l}7.22 \\
(1.62)\end{array}$ & $\begin{array}{l}137.86 \\
(24.28)\end{array}$ \\
\hline & $8(21)$ & 30791 & $\begin{array}{l}0.79 \\
(0.10)\end{array}$ & $\begin{array}{l}1.09 \\
(0.10)\end{array}$ & $\begin{array}{l}8.12 \\
(0.93)\end{array}$ & $\begin{array}{l}0.56 \\
(0.08)\end{array}$ & $\begin{array}{l}0.76 \\
(0.06)\end{array}$ & $\begin{array}{l}5.89 \\
(0.72)\end{array}$ & $\begin{array}{l}108.0 \\
(11.61)\end{array}$ \\
\hline \multirow{3}{*}{$\begin{array}{l}\text { North } \\
\text { Atlantic }\end{array}$} & 4 4(9) & 9782 & $\begin{array}{l}1.28 \\
(0.12)\end{array}$ & $\begin{array}{l}2.42 \\
(0.24)\end{array}$ & $\begin{array}{l}15.10 \\
(4.20)\end{array}$ & $\begin{array}{l}0.84 \\
(0.04)\end{array}$ & $\begin{array}{l}1.47 \\
(0.11)\end{array}$ & $\begin{array}{l}10.47 \\
(3.40)\end{array}$ & $\begin{array}{l}174.51 \\
(10.68)\end{array}$ \\
\hline & $6(15)$ & 8999 & $\begin{array}{l}1.17 \\
(0.15)\end{array}$ & $\begin{array}{l}2.13 \\
(0.32)\end{array}$ & $\begin{array}{l}10.30 \\
(1.26)\end{array}$ & $\begin{array}{l}0.79 \\
(0.08)\end{array}$ & $\begin{array}{l}1.32 \\
(0.14)\end{array}$ & $\begin{array}{l}6.58 \\
(0.96)\end{array}$ & $\begin{array}{l}161.74 \\
(15.36)\end{array}$ \\
\hline & $8(21)$ & 8276 & $\begin{array}{l}1.05 \\
(0.04)\end{array}$ & $\begin{array}{l}2.10 \\
(0.34)\end{array}$ & $\begin{array}{l}10.69 \\
(1.14)\end{array}$ & $\begin{array}{l}0.71 \\
(0.03)\end{array}$ & $\begin{array}{l}1.38 \\
(0.13)\end{array}$ & $\begin{array}{l}7.42 \\
(0.98)\end{array}$ & $\begin{array}{l}158.92 \\
(12.62)\end{array}$ \\
\hline
\end{tabular}

hours at a temporal resolution of 6 hours for track prediction with a lead time of 24 hours. A comparison with our model with $T=8$ is provided in Table 3 . One can see that we have achieved better results for a much harder problem with arbitrary lead time depending on the time between TC's initiation and its landfall. The authors of [13] have also reported the error range (std) $71 \mathrm{KM}$ for a subset of cyclones in the Atlantic ocean, while our model achieves an error range (std) $2.87 \mathrm{KM}-22.44 \mathrm{KM}$ across all ocean basins. Also, the authors of [13] reported average performance on the test set for three runs of the model, while we have reported 5-fold performance of our model. All this makes our model more robust and reliable to use for practical purposes.

In [14] authors use a combination of historical TC data, reanalysis data, and output from operational models and propose eight models for track prediction of TCs in NA and EP ocean basins for years 2017 to 2019. The model which close to our proposed model is HURR-(viz, CNN/GRU) which uses CNN-encoder and GRU-decoder [41]. The authors consider only those hurricanes in their study for which MSWS reaches 34 knots at some time $t_{0}$ and contains at least $60 \mathrm{~h}$ of data after $t_{0}$. Our model does not have any such restrictions. In Table 4 , we have shown the results for HURR-(viz, CNN/GRU) and the operational CLP5 model for EP and NA ocean basin. One can see that our results are not as good as that of HURR-(viz, CNN/GRU) but quite comparable with the operational model CLP5. One can notice that the standard deviation of our model is quite low in comparison to these two models. Here, we would 


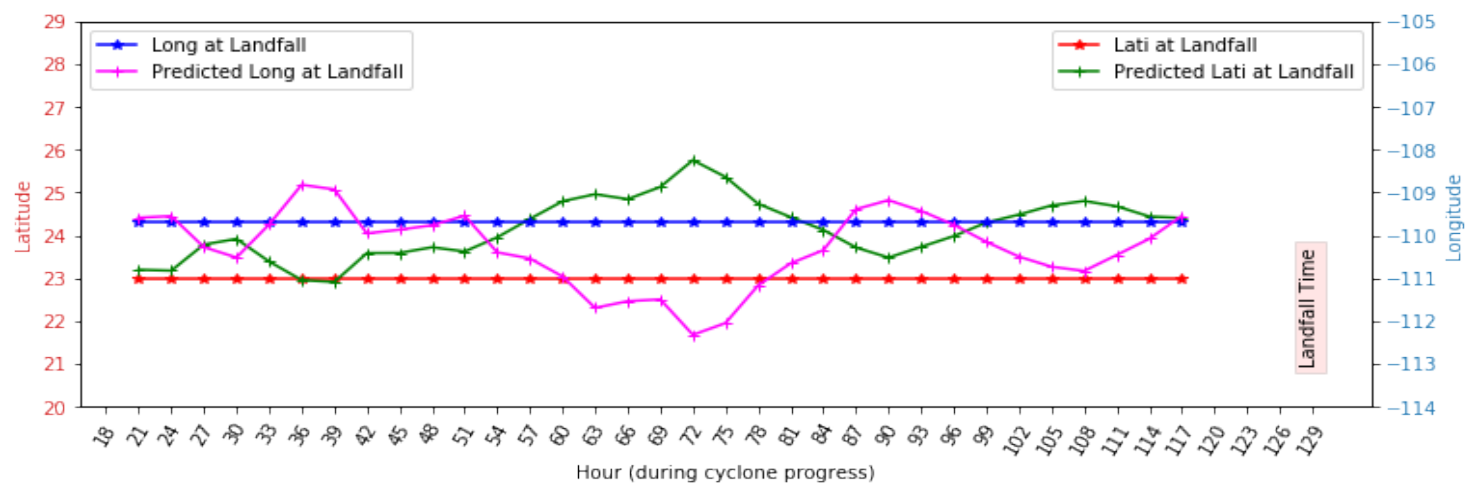

Figure 6: Latitude and Longitude prediction for hurricane BUD (2018) in East Pacific ocean for T = 8

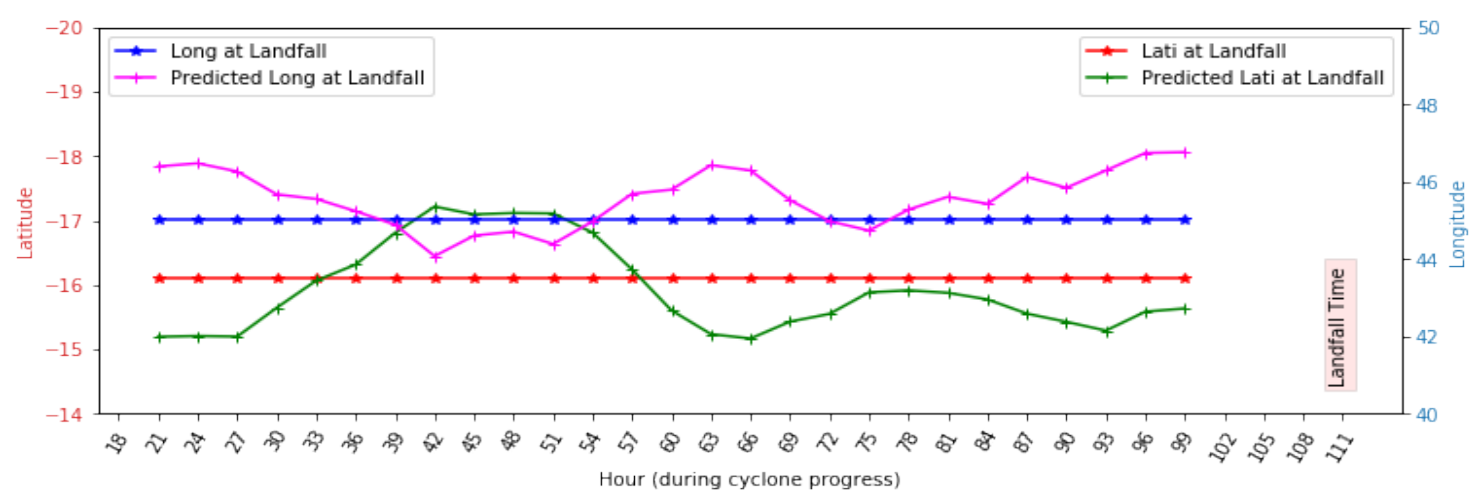

Figure 7: Latitude and Longitude prediction for cyclone BELNA (2019) in South Indian ocean for T = 8

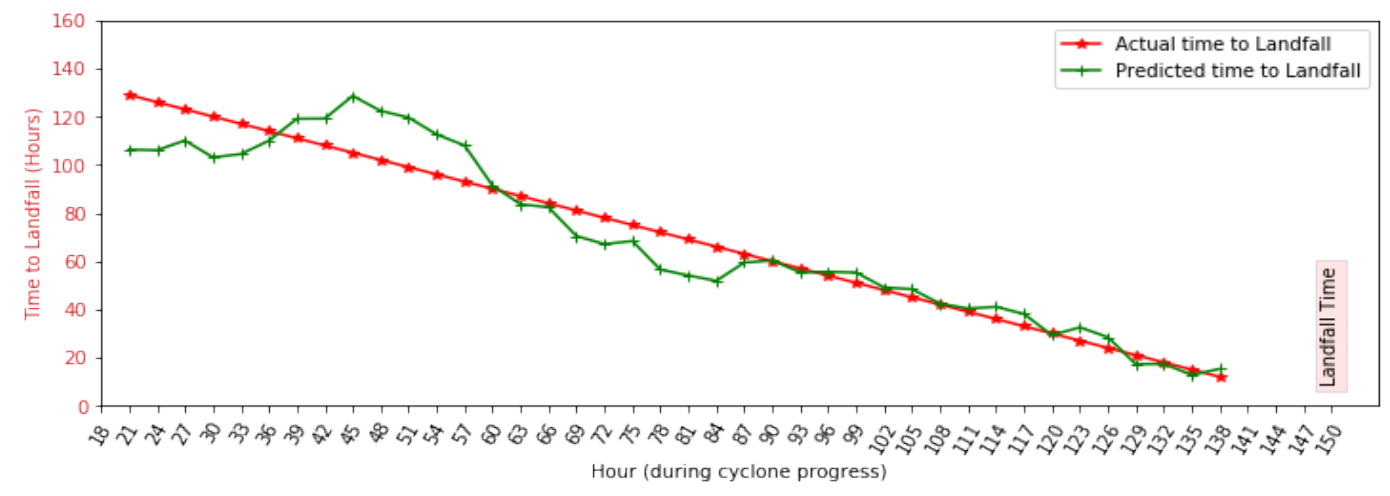

Figure 8: Time to Landfall prediction for Fani (2019) cyclone in North Indian ocean for T = 8

again point out that we are not making a direct comparison here as our target prediction problem is different from the above-mentioned works but, at the same time, much more challenging and important.

We do not find any meteorological department across the world which reported the landfall prediction accuracy except the Indian Meteorological Department (IMD) on its website [42]. IMD has reported landfall's location error and time for a certain number of lead hours. In Table 5, we have reported the last 4/5 years (as per data availability) MAE achieved by IMD for landfall's location and time prediction. From Table 1 , we can see that in the NI ocean basin, the landfall occurs on average at 95.61 hours. Therefore, it is reasonable to compare our results with that of IMD for 72 lead hours. Clearly, here also, our model performs better than that of models used by IMD for both landfall's location and time. We have not included the results reported by IMD for earlier years, as errors are much higher. 


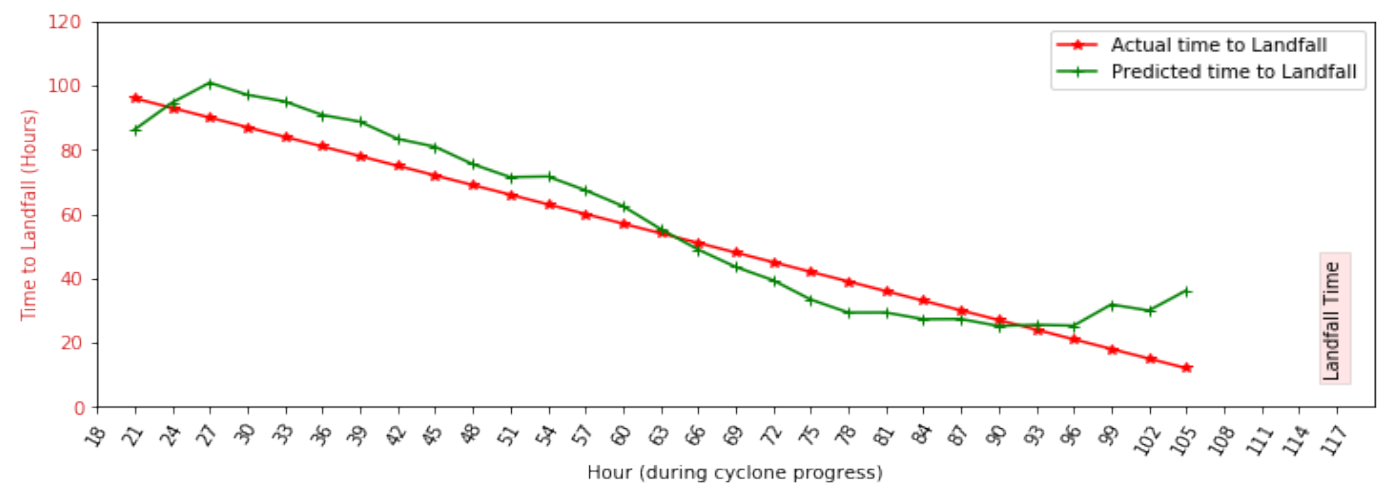

Figure 9: Time to Landfall prediction for NAKRI (2019) cyclone in West Pacific ocean for $\mathrm{T}=8$

Table 3: Comparison in terms of distance MAE (KM)

\begin{tabular}{|l|l|l|l|l|l|l|l|l|l|l|}
\hline $\begin{array}{l}\text { Model /Ocean } \\
\text { Basin }\end{array}$ & $\begin{array}{l}\text { NI } \\
(\mathrm{KM})\end{array}$ & $\begin{array}{l}\text { SI } \\
(\mathrm{KM})\end{array}$ & $\begin{array}{l}\mathrm{EP} \\
(\mathrm{KM})\end{array}$ & $\begin{array}{l}\text { SP } \\
(\mathrm{KM})\end{array}$ & $\begin{array}{l}\text { WP } \\
(\mathrm{KM})\end{array}$ & $\begin{array}{l}\text { NA } \\
(\mathrm{KM})\end{array}$ & $\begin{array}{l}\text { No. of pa- } \\
\text { rameters } \\
\left(* 10^{6}\right)\end{array}$ & $\begin{array}{l}\text { Training } \\
\text { Time }\end{array}$ & Lead Time & $\begin{array}{l}\text { Spacial } \\
\text { Extent } \\
(\text { Resolu- } \\
\text { tion) }\end{array}$ \\
\hline $\begin{array}{l}\text { Fusion } \\
\text { (Track) [13] }\end{array}$ & 138.9 & 136.1 & 106.9 & 161.7 & 136.1 & 130.2 & $\geq 2.27$ & $8 \mathrm{~h}$ & $24 \mathrm{~h}$ & $\begin{array}{l}25^{\circ} \times 25^{\circ} \\
\left(1^{\circ} \times 1^{\circ}\right)\end{array}$ \\
\hline $\begin{array}{l}\text { Proposed } \\
\text { Model (Land- } \\
\text { fall) }\end{array}$ & 66.1 & 119.9 & 110.4 & 144.8 & 108.0 & 158.9 & 0.157 & $\begin{array}{l}\leq \\
0.75 \mathrm{~h}\end{array}$ & $\begin{array}{l}\text { TC depen- } \\
\text { dent }(>= \\
12 h)\end{array}$ & $\begin{array}{l}4^{\circ} \times 4^{\circ} \\
\left(0.25^{\circ} \times\right. \\
\left.0.25^{\circ}\right)\end{array}$ \\
\hline
\end{tabular}

Table 4: Track prediction MAE (std) in KM for HURR model and operational CLP5 model for 24 hour lead time

\begin{tabular}{|l|l|l|l|l|}
\hline \multirow{2}{*}{$\mathbf{E P}$} & Model/Year & $2017(10 \mathrm{TCs})$ & $2018(15 \mathrm{TCs})$ & $2019(12 \mathrm{TCs})$ \\
\cline { 2 - 5 } & HURR-(viz, cnn/gru) & $74(40)$ & $69(42)$ & $73(45)$ \\
\cline { 2 - 5 } NA & HUP & $114(59)$ & $109(61)$ & $133(74)$ \\
\cline { 2 - 5 } & CLP5 & $113(77)$ & $123(89)$ \\
\hline
\end{tabular}

Table 5: 4/5 year (2015-2019) MAE reported by IMD for cyclones in NI ocean basin

\begin{tabular}{|l|l|l|l|l|}
\hline Lead Time (hours) & 36 & 48 & 60 & 72 \\
\hline Landfall Time & 4.96 & 5.53 & 6.8 & 9.6 \\
\hline Landfall Distance & 42.84 & 78.08 & 92.6 & 112.5 \\
\hline
\end{tabular}

\section{Conclusion}

We propose a model that can predict the landfall's location and time of a TC with high accuracy by observing a TC for $9 \mathrm{~h}, 15 \mathrm{~h}$, or $21 \mathrm{~h}$ at any time of its progression in the world's six ocean basins. The model took only 30 to 45 minutes for training and can predict the landfall characteristics within few seconds, which makes our model suitable for practical usage where the disaster managers can know the landfall location and time well in advance and can take preventive life saving and property saving measures well in time. Our model supports the case that deep learning models like CNN and LSTM can be utilized to predict challenging and complex prediction problems like the landfall of a TC. One can further work in the direction of utilizing CNN with Attention and Transformers models for further improvement. One can also develop Consensus models to solve the proposed prediction problem. As we are able to solve a complex landfall prediction problem using data over a small spatial extent with high resolution, it will be interesting to see to use the same kind of data for track or intensity prediction problems. 


\section{References}

[1] Aslak Grinsted, Peter Ditlevsen, and Jens Hesselbjerg Christensen. Normalized us hurricane damage estimates using area of total destruction, 1900-2018. Proceedings of the National Academy of Sciences, 116(48):2394223946, 2019.

[2] Christian Webersik, Miguel Esteban, and Tomoya Shibayama. The economic impact of future increase in tropical cyclones in japan. Natural Hazards, 55:233-250, 112010.

[3] Marie-Dominique Leroux, Kimberly Wood, Russell L. Elsberry, Esperanza O. Cayanan, Eric Hendricks, Matthew Kucas, Peter Otto, Robert Rogers, Buck Sampson, and Zifeng Yu. Recent advances in research and forecasting of tropical cyclone track, intensity, and structure at landfall. Tropical Cyclone Research and Review, 7(2):85 - 105, 2018.

[4] NOAA. https://www.nhc.noaa.gov/modelsummary.shtml, 2019.

[5] T. M. Hall and S. Jewson. Statistical modeling of north atlantic tropical cyclone tracks. Tellus, 59A:486-498, 2007.

[6] T. N. Krishnamurti, C. M. Kishtawal, Zhan Zhang, Timothy LaRow, David Bachiochi, Eric Williford, Sulochana Gadgil, and Sajani Surendran. Multimodel ensemble forecasts for weather and seasonal climate. Journal of Climate, 13(23):4196 - 4216, 2000.

[7] Mina Moradi Kordmahalleh, Mohammad Gorji Sefidmazgi, and Abdollah Homaifar. A sparse recurrent neural network for trajectory prediction of atlantic hurricanes. GECCO '16, page 957-964, New York, NY, USA, 2016. Association for Computing Machinery.

[8] Sutapa Chaudhuri, Debjani Basu, D. Das, Sayantika Goswami, and S. Varshney. Swarm intelligence and neural nets in forecasting the maximum sustained wind speed along the track of tropical cyclones over bay of bengal. Natural Hazards, 87, 072017.

[9] Rita Kovordányi and Chandan Roy. Cyclone track forecasting based on satellite images using artificial neural networks. ISPRS Journal of Photogrammetry and Remote Sensing, 64(6):513 - 521, 2009.

[10] Sookyung Kim, Hyojin Kim, Joonseok Lee, Sangwoong Yoon, Samira Ebrahimi Kahou, Karthik Kashinath, and Mr Prabhat. Deep-hurricane-tracker: Tracking and forecasting extreme climate events. pages 1761-1769, 01 2019.

[11] Sheila Alemany, Jonathan Beltran, Adrian Perez, and Sam Ganzfried. Predicting hurricane trajectories using a recurrent neural network. Proceedings of the AAAI Conference on Artificial Intelligence, 33, 022018.

[12] Song Gao, Peng Zhao, Bin Pan, Yaru Li, Min Zhou, Jiangling Xu, Shan Zhong, and Zhenwei Shi. A nowcasting model for the prediction of typhoon tracks based on a long short term memory neural network. Acta Oceanologica Sinica, 37:8-12, 052018.

[13] Sophie Giffard-Roisin, Mo Yang, Guillaume Charpiat, Christina Kumler Bonfanti, Balázs Kégl, and Claire Monteleoni. Tropical cyclone track forecasting using fused deep learning from aligned reanalysis data. Frontiers in Big Data, 3:1, 2020.

[14] Léonard Boussioux, Cynthia Zeng, Théo Guénais, and Dimitris Bertsimas. Hurricane forecasting: A novel multimodal machine learning framework, 2020.

[15] Rui Chen, Xiang Wang, Weimin Zhang, Xiaoyu Zhu, Aiping Li, and Chao Yang. A hybrid cnn-lstm model for typhoon formation forecasting. 23(3):375-396, July 2019.

[16] P Berrisford and Coauthors. The era-interim archive version 2.0. (1):23, 112011.

[17] European Centre for Medium-Range Weather Forecasts. Era5 reanalysis, 2017.

[18] Kenneth R. Knapp, Michael C. Kruk, David H. Levinson, Howard J. Diamond, and Charles J. Neumann. The international best track archive for climate stewardship (ibtracs): Unifying tropical cyclone data. Bulletin of the American Meteorological Society, 91(3):363 - 376, 01 Mar. 2010.

[19] IBTrACS. https://www.ncdc.noaa.gov/ibtracs/index.php?name=ib-v4-access, 2020.

[20] Warren S McCulloch and Walter Pitts. A logical calculus of the ideas immanent in nervous activity. The bulletin of mathematical biophysics, 5(4):115-133, 1943.

[21] J. Kiefer and J. Wolfowitz. Stochastic estimation of the maximum of a regression function. Ann. Math. Statist., 23(3):462-466, 091952.

[22] Michael I. Jordan. Attractor Dynamics and Parallelism in a Connectionist Sequential Machine, page $112-127$. IEEE Press, 1990. 
[23] Axel Cleeremans, David Servan-Schreiber, and James L. McClelland. Finite state automata and simple recurrent networks. Neural Comput., 1(3):372-381, September 1989.

[24] Pearlmutter. Learning state space trajectories in recurrent neural networks. In International 1989 Joint Conference on Neural Networks, pages 365-372 vol.2, 1989.

[25] Y. Bengio, P. Simard, and P. Frasconi. Learning long-term dependencies with gradient descent is difficult. IEEE Transactions on Neural Networks, 5(2):157-166, 1994.

[26] Sepp Hochreiter and Jürgen Schmidhuber. Long short-term memory. Neural Comput., 9(8):1735-1780, November 1997.

[27] F. A. Gers, J. Schmidhuber, and F. Cummins. Learning to forget: continual prediction with 1stm. In 1999 Ninth International Conference on Artificial Neural Networks ICANN 99. (Conf. Publ. No. 470), volume 2, pages 850-855 vol.2, 1999.

[28] Felix A. Gers, Nicol N. Schraudolph, and Jürgen Schmidhuber. Learning precise timing with lstm recurrent networks. J. Mach. Learn. Res., 3(null):115-143, March 2003.

[29] F. A. Gers and E. Schmidhuber. Lstm recurrent networks learn simple context-free and context-sensitive languages. IEEE Transactions on Neural Networks, 12(6):1333-1340, 2001.

[30] Y. LeCun, B. Boser, J. S. Denker, D. Henderson, R. E. Howard, W. Hubbard, and L. D. Jackel. Backpropagation applied to handwritten zip code recognition. Neural Computation, 1(4):541-551, 1989.

[31] Alex Krizhevsky, Ilya Sutskever, and Geoffrey Hinton. Imagenet classification with deep convolutional neural networks. Neural Information Processing Systems, 25, 012012.

[32] F. Milletari, N. Navab, and S. Ahmadi. V-net: Fully convolutional neural networks for volumetric medical image segmentation. In 2016 Fourth International Conference on 3D Vision (3DV), pages 565-571, 2016.

[33] Mayur Mudigonda, Sookyung Kim, Ankur Mahesh, Samira Kahou, Karthik Kashinath, Dean Williams, Vincen Michalski, Travis O'Brien, and Mr Prabhat. Segmenting and tracking extreme climate events using neural networks. In Deep Learning for Physical Sciences (DLPS) Workshop, held with NIPS Conference, 2017.

[34] Emmanuel de Bézenac, Arthur Pajot, and Patrick Gallinari. Deep learning for physical processes: incorporating prior scientific knowledge. Journal of Statistical Mechanics: Theory and Experiment, 2019(12):124009, dec 2019.

[35] Hui Su, Longtao Wu, Jonathan H. Jiang, Raksha Pai, Alex Liu, Albert J. Zhai, Peyman Tavallali, and Mark DeMaria. Applying satellite observations of tropical cyclone internal structures to rapid intensification forecast with machine learning. Geophysical Research Letters, 47(17):e2020GL089102, 2020. e2020GL089102 2020GL089102.

[36] François Chollet. Keras. https://github.com/fchollet/keras, 2015.

[37] Martín Abadi, Ashish Agarwal, Paul Barham, Eugene Brevdo, Zhifeng Chen, Craig Citro, Greg S. Corrado, Andy Davis, Jeffrey Dean, Matthieu Devin, Sanjay Ghemawat, Ian Goodfellow, Andrew Harp, Geoffrey Irving, Michael Isard, Yangqing Jia, Rafal Jozefowicz, Lukasz Kaiser, Manjunath Kudlur, Josh Levenberg, Dandelion Mané, Rajat Monga, Sherry Moore, Derek Murray, Chris Olah, Mike Schuster, Jonathon Shlens, Benoit Steiner, Ilya Sutskever, Kunal Talwar, Paul Tucker, Vincent Vanhoucke, Vijay Vasudevan, Fernanda Viégas, Oriol Vinyals, Pete Warden, Martin Wattenberg, Martin Wicke, Yuan Yu, and Xiaoqiang Zheng. TensorFlow: Large-scale machine learning on heterogeneous systems, 2015. Software available from tensorflow.org.

[38] F. Pedregosa, G. Varoquaux, A. Gramfort, V. Michel, B. Thirion, O. Grisel, M. Blondel, P. Prettenhofer, R. Weiss, V. Dubourg, J. Vanderplas, A. Passos, D. Cournapeau, M. Brucher, M. Perrot, and E. Duchesnay. Scikit-learn: Machine learning in Python. Journal of Machine Learning Research, 12:2825-2830, 2011.

[39] Diederik Kingma and Jimmy Ba. Adam: A method for stochastic optimization. International Conference on Learning Representations, 122014.

[40] Vinod Nair and Geoffrey E. Hinton. Rectified linear units improve restricted boltzmann machines. In Johannes Fürnkranz and Thorsten Joachims, editors, Proceedings of the 27th International Conference on Machine Learning (ICML-10), June 21-24, 2010, Haifa, Israel, pages 807-814. Omnipress, 2010.

[41] Junyoung Chung, Çaglar Gülçehre, KyungHyun Cho, and Yoshua Bengio. Empirical evaluation of gated recurrent neural networks on sequence modeling. CoRR, abs/1412.3555, 2014.

[42] RMSC. http://www.rsmcnewdelhi.imd.gov.in/landfall-forecast.php, 2020. 